\title{
Oral glutamine in the prevention of fluorouracil induced intestinal toxicity: a double blind, placebo controlled, randomised trial
}

B Daniele, F Perrone, C Gallo, S Pignata, S De Martino, R De Vivo, E Barletta, R Tambaro, R Abbiati, L D'Agostino
Systemic chemotherapy produces changes in the structure of the intestinal mucosa ${ }^{1-3}$ that are associated with increased permeability of the intestine ${ }^{4}$ and probably with an increased risk of bacteraemia and endotoxaemia. 5-Fluorouracil (FU) induces mitotic arrest of intestinal crypt cells resulting in an increased ratio of crypt cells to villous enterocytes and thus a reduction of the absorptive surface. ${ }^{15}$ The clinical counterpart of these changes is diarrhoea, observed in up to $50 \%$ of patients ${ }^{6} 7$ treated with FU and folinic acid (FA), a widely used combination for the treatment of colorectal cancer. ${ }^{8}{ }^{9}$ Together with stomatitis that may develop in $60 \%$ of patients receiving $\mathrm{FU} / \mathrm{FA},{ }^{10}$ diarrhoea can have a major impact on the patient's quality of life.

Glutamine is the most abundant free amino acid in the body. ${ }^{11}$ In several animal species, glutamine was shown to be the major respiratory fuel for the intestinal tract. ${ }^{12-14}$ Moreover, reduction of plasma glutamine levels by administration of glutaminase caused oedema and ulceration of the intestinal mucosa as well as patchy areas of necrosis. ${ }^{15}$ In the rat, oral glutamine reduced the severity of enterocolitis induced by toxic doses of methotrexate ${ }^{16}$ and radiation induced intestinal mucosal injury. ${ }^{17}$ Depletion of the glutamine pool caused epithelial atrophy in the small intestine which may be associated with breakdown of the gut barrier and facilitated bacterial translocation. ${ }^{18}$

Following demonstration that parenteral administration of glutamine had protective effects on the intestinal mucosa under different conditions, ${ }^{19}{ }^{20}$ we hypothesised that oral supplements of glutamine could prevent intestinal toxicity in patients receiving chemotherapy with FU/FA.

As intestinal toxicity by $\mathrm{FU}$ is associated with changes in intestinal absorption (IA $)^{21}$ and permeability (IP), ${ }^{4}$ we conducted a randomised pilot study to evaluate if oral glutamine could help prevent the changes in IA and IP induced by chemotherapy with FU/FA in patients with colorectal cancer. The D-xylose absorption test and the cellobiose-mannitol permeability test were used to assess IA and IP, respectively. These tests are sensitive and reliable and have proved useful in many clinical conditions characterised by disruption of the normal architecture of the small intestinal mucosa, such as in coeliac disease and Crohn's disease. ${ }^{22-24}$

Abbreviations used in this paper: FU, 5-fluorouracil; FA, folinic acid; IP, intestinal permeability; IA, intestinal absorption; AUC, area under the curve; PS, performance status. 


\section{Patients and methods}

We conducted a double blind, two arm, parallel, randomised controlled trial comparing oral glutamine with placebo.

Seventy patients entered the study between June 1996 and April 1998. They were scheduled to receive chemotherapy with FU/FA as treatment for advanced or metastatic colon cancer (the so called "advanced" setting) or as adjuvant therapy, as a precautionary adjunctive treatment after surgical resection for colon cancer. All patients had a performance status (PS) not worse than 2 according to the Eastern Cooperative Oncologic Group scale (PS $0=$ normal activity; PS $1=$ symptoms but fully ambulatory; PS 2=some bed rest, but in bed less than $50 \%$ of normal daytime). Chemotherapy consisted of daily administration of $100 \mathrm{mg} / \mathrm{m}^{2}$ FA followed by $450 \mathrm{mg} / \mathrm{m}^{2} \mathrm{FU}$ for five days. FA was given by intravenous infusion over 30 minutes followed by an intravenous bolus dose of FU between 9 and $11 \mathrm{am}$. Patients had not received chemotherapy previously. Creatinine clearance was normal. Patients gave informed consent to participate in the study which was approved by the National Cancer Institute ethics committee.

Glutamine and placebo were obtained as crystalline powders from Bracco Pharmaceutical Company, in sachets, each containing $3 \mathrm{~g}$ of either glutamine or placebo (maltodestrins). The organoleptic features of glutamine and placebo as well as their appearance were identical. Patients were instructed to consume the contents of six sachets $(18 \mathrm{~g}) /$ day dissolved in water. The suggested administration schedule was two sachets $(6 \mathrm{~g})$ three times daily. No specific relation with meals was suggested. To assess compliance, patients were instructed to record the number of sachets consumed each day and to return unused sachets. Glutamine or placebo was administered for 15 consecutive days, starting five days before the first day of chemotherapy. The study was limited to the first cycle of chemotherapy.

Baseline evaluations of IA and IP were performed two and one day before starting the study treatment, respectively. Post-treatment IA and IP tests were performed one and two days after stopping the study treatment.

To obtain data on toxicity, patients were provided with a diary to record the following information daily: nausea (yes/no); vomiting (yes/no; number of episodes); number of stools; consistency of stools (normal; soft; watery); presence of faecal blood (yes/no); abdominal pain (yes/no); and number of loperamide tablets consumed. In the event of diarrhoea, patients were instructed to take loperamide tablets ( $2 \mathrm{mg}$ ) as follows: a $4 \mathrm{mg}$ dose after the first watery stool followed by $2 \mathrm{mg}$ doses every four hours. Two weeks after the start of chemotherapy a complete blood cell count and routine biochemistry were performed. Toxicity of chemotherapy was graded according to the common toxicity criteria of the National Cancer Institute..$^{25}$ For diarrhoea, common toxicity criteria grades were defined as follows: grade 0 , none; grade 1 , increase of $<4$ stools/day over pretreatment; grade 2, increase of 4-6 stools/day or nocturnal stools; grade 3 , increase of $\geqslant 7$ stools/day, incontinence, or need for parenteral support for dehydration; grade 4, physiological consequences requiring intensive care or haemodynamic collapse.

D-XYLOSE ABSORPTION TEST

After an overnight fast (6-8 hours), patients ingested a $25 \mathrm{~g}$ dose of D-xylose (Sigma, Milan, Italy) and were encouraged to drink water to promote diuresis. Urine was collected for the next five hours. Two aliquots were stored at $-25^{\circ} \mathrm{C}$ and assayed within five days. ${ }^{26}$ Urinary recovery of $\mathrm{D}$-xylose was calculated as a percentage of the dose ingested.

CELLOBIOSE-MANNITOL PERMEABILITY TEST After an overnight fast (6-8 hours), the sugars (5 g cellobiose+2 g mannitol) (Sigma) dissolved in $100 \mathrm{ml}$ of water were ingested. This solution has an osmolality of approximately 270 mosmol. A baseline urine specimen was obtained. After one hour patients were encouraged to drink water to promote diuresis. Urine passed within five hours from the beginning of the test was collected, the volume measured, and two aliquots stored immediately at $-25^{\circ} \mathrm{C}$ and assayed within five days. Before mannitol and cellobiose assays, the presence of urinary glucose, which could interfere with the results, was tested using the Ketodiabur strip test (Boehringer Mannheim, Italy); all urine tested was free of glucose. Urinary mannitol was determined according to the method of Corcoran and Page $^{27}$ and urinary cellobiose was assayed according to Strobel and colleagues. ${ }^{28}$ Urinary recovery of both cellobiose and mannitol was calculated as a percentage of the dose ingested. Cellobiose is minimally absorbed in the small intestine across paracellular tight junctions while mannitol is thought to be absorbed by transcellular pathways through aqueous pores in the enterocyte brush border. ${ }^{22}$ When intestinal damage is present, recovery of cellobiose increases and that of mannitol decreases. The ratio between the percentages of cellobiose and mannitol recovered - that is, a further measure of mucosal integrity - was also calculated. ${ }^{28}$

\section{STATISTICAL METHODS}

Sample size was estimated using D-xylose absorption as the primary end point. Based on a previous report, ${ }^{20}$ we targeted the study to have a power of $90 \%$ to recognise an effect size of 0.8 -that is, a difference between mean values of $\mathrm{D}$-xylose absorption equal to $80 \%$ of the standard deviation. Approximately 70 patients were needed (Solo Statistical System Power Analysis, BMDP Statistical Software, Cork, Ireland, 1991). Patients were randomised by telephoning the clinical trials office where clinicians used a computer driven procedure with stage of therapy (adjuvant/ advanced) as the stratifying variable. Packages containing the anonymous supply of treatment (glutamine or placebo) for each patient were provided by the clinical trials office. Patients, clinicians, and the statistician were blind to the 
Table 1 Baseline characteristics of patients in the glutamine and placebo groups

\begin{tabular}{lcc}
\hline & Glutamine $(n=29)$ & Placebo $(n=33)$ \\
\hline Sex (n (\%)) & & \\
$\quad$ Males & $17(58.6)$ & $19(57.6)$ \\
$\quad$ Females & $12(41.4)$ & $14(42.4)$ \\
Age (median, range) & $63(44-76)$ & $61(35-75)$ \\
Treatment setting (n (\%)) & & \\
$\quad$ Adjuvant & $8(27.6)$ & $10(30.3)$ \\
$\quad$ Advanced & $21(72.4)$ & $23(69.7)$ \\
Performance status (n (\%)) & $20(69.0)$ & $22(66.7)$ \\
0 & $6(20.7)$ & $8(24.2)$ \\
1 & $3(10.3)$ & $3(9.1)$ \\
2 & $22.8(2.5)$ & $23.2(2.6)$ \\
Body mass index (mean (SD)) &
\end{tabular}

Table 2 Absorption and permeability test values before and after the experimental treatment in the glutamine and placebo groups

\begin{tabular}{lccc}
\hline & Glutamine (n=29) & Placebo (n=33) & p Value \\
\hline D-Xylose (mean (SD) \% of dose ingested) & & & \\
Before treatment & $20.3(3.4)$ & $20.6(4.7)$ & \\
After treatment & $16.6(4.8)$ & $13.5(3.5)$ & 0.02 \\
Before-after experimental treatment & $3.8(4.7)$ & $7.1(5.3)$ & \\
Mannitol (mean (SD) \% of dose ingested) & $21.5(7.0)$ & $21.2(7.2)$ & \\
Before treatment & $16.9(6.6)$ & $12.0(5.5)$ & \multirow{2}{*}{0.02} \\
After treatment & $4.5(7.7)$ & $9.2(6.2)$ & \\
Before-after experimental treatment & & $0.28(0.18)$ & \\
Cellobiose (mean (SD) \% of dose ingested) & $0.27(0.21)$ & $0.45(0.34)$ & \multirow{2}{*}{0.60} \\
Before treatment & $0.37(0.27)$ & $-0.17(0.31)$ & \\
After treatment & $-0.10(0.34)$ & & \\
Before-after experimental treatment & & & \\
Cellobiose/mannitol ratio (mean (SD)) & $0.015(0.013)$ & $0.015(0.012)$ & \\
Before treatment & $0.027(0.023)$ & $0.052(0.046)$ & \multirow{2}{*}{0.04} \\
After treatment & $-0.012(0.024)$ & $-0.037(0.044)$ & \\
Before-after experimental treatment & &
\end{tabular}

${ }^{\star}$ Mann-Whitney test

assigned treatment. Overall effect of chemotherapy on IA and IP was assessed by comparing pre- and post-treatment values for the whole group using the Wilcoxon signed rank test. For each patient, differences between IP and IA tests before and after treatment were calculated. Differences were then compared between the two study arms by the nonparametric Mann-Whitney test. Daily assess- ments of diarrhoea were summarised for each subject by means of AUC using the trapezoidal rule. ${ }^{29}$ All $\mathrm{p}$ values were two sided.

\section{Results}

Sixty two patients were evaluable for analysis: 29 in the glutamine and 33 in the placebo arm. Eight patients (six in the glutamine and two in the placebo arm) were excluded from analysis because they did not perform the posttreatment functional assessment. Of these, administration of study treatment was not completed because of severe heartburn (one case), myocardial infarction (one case), severe stomatitis (two cases), intense nausea (one case), and emergency surgery (one case); one patient refused treatment soon after randomisation and one patient was excluded because he erroneously received chemotherapy at a dosage lower than planned.

Table 1 shows the baseline characteristics of the 62 evaluable patients. There were no differences in baseline clinical and functional characteristics between the two arms. Only seven patients $(11 \%)$ were severely malnourished with a body mass index $<20 \mathrm{~kg} / \mathrm{m}^{2}$. There was no difference between the placebo and glutamine arms in mean percentage of sachets consumed ( $88 \%$ and $87 \%$, respectively).

TREATMENT EFFECT ON IA AND IP

For the whole group, chemotherapy induced significant worsening of IA and IP. Mean values for D-xylose and mannitol absorption decreased by absolute values of $5.5 \%$ (relative decrease $23 \% ; \mathrm{p}<0.0001$ ) and $7.0 \%$ (relative decrease $33 \%$; $<<0.0001$ ), respectively; mean values for cellobiose absorption and the cellobiose/mannitol ratio increased by absolute values of $0.14 \%$ (relative increase $50 \%$;
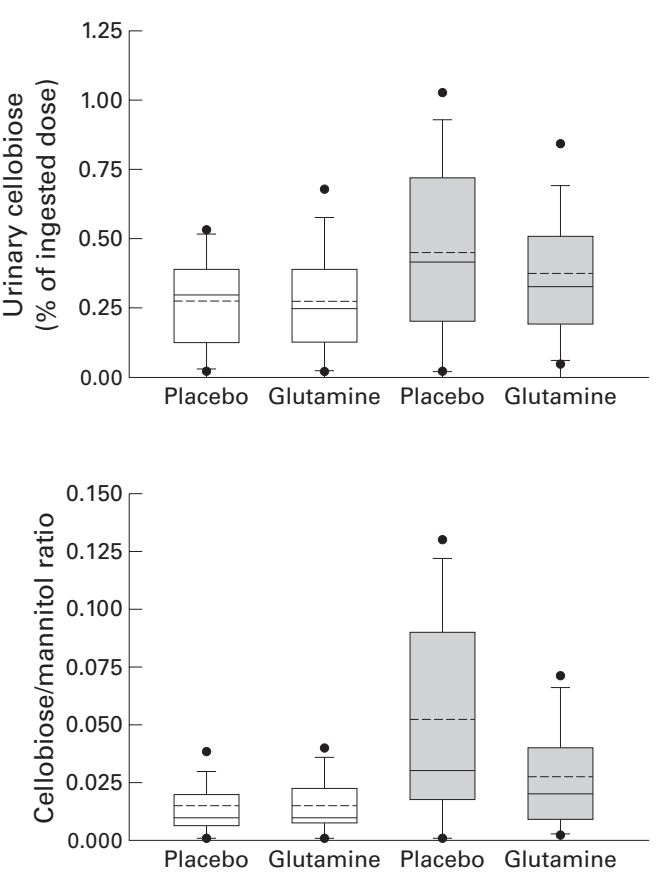

Figure 1 Box plots of pre-(open boxes) and post-(shaded boxes) treatment values for intestinal absorption and intestinal permeability parameters. Solid lines indicate the 5th, 25th, 50th, 75th, and 95th percentiles; broken lines indicate mean values; solid dots indicate upper and lower values. 
Table 3 Incidence of different grades of diarrhoea in the glutamine and placebo groups according to the criteria of the National Cancer Institute

\begin{tabular}{lll}
\hline & No (\%) of patients \\
\cline { 2 - 3 } & Glutamine $(n=29)$ & Placebo $(n=33)$ \\
\hline Grade 0 & $17(58.6)$ & $14(42.4)$ \\
Grade 1 & $5(17.2)$ & $10(30.3)$ \\
Grade 2 & $4(13.8)$ & $6(18.2)$ \\
Grade 3 & $3(10.3)$ & $2(6.1)$ \\
Grade 4 & - & $1(3.0)$ \\
\hline
\end{tabular}

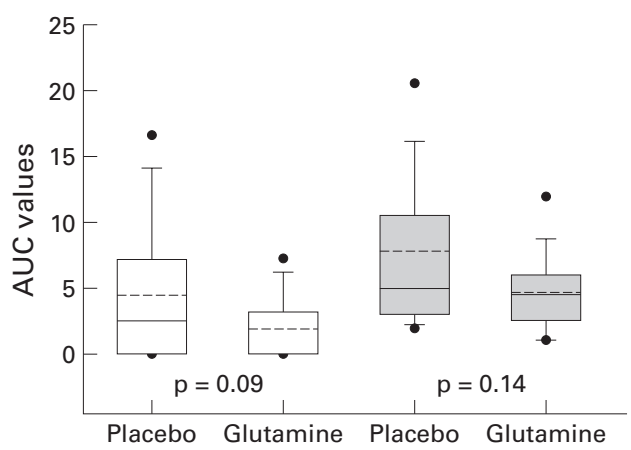

Figure 2 Box plots of area under the curve (AUC) values by treatment arm for all patients (open boxes) and for those with diarrhoea (shaded boxes). Solid lines indicate the 5th, 25 th, 50th, 75th, and 95th percentiles; broken lines indicate mean values; solid dots indicate upper and lower values.

$\mathrm{p}=0.003$ ) and 0.025 (relative increase $167 \%$; $\mathrm{p}<0.0001)$, respectively.

Table 2 shows pretreatment, post-treatment, and the difference between these values for IA and IP tests by treatment arm. Reduction in IA (D-xylose absorption) was more marked in the placebo arm $(7.1 \% v 3.8 \%$; $=0.02)$. Reduction in IP to mannitol was similarly higher in the placebo arm $(9.2 \% v 4.5 \% ; \mathrm{p}=0.02)$. In contrast, urinary recovery of cellobiose was not different between the study arms $(p=0.60)$. Accordingly, the cellobiose/mannitol ratio increased more in the placebo arm (0.037 v $0.012 ; \mathrm{p}=0.04)$. Baseline and post-treatment distributions of the functional tests are reported in fig 1 .

\section{TREATMENT EFFECT ON TOXICITY OF}

CHEMOTHERAPY

Table 3 shows the incidence of different grades of diarrhoea according to treatment arm. One case of grade 4 diarrhoea was observed in the placebo and none in the glutamine arm. Diarrhoea lasted longer in patients receiving placebo compared with glutamine. Also, the AUC for diarrhoea was larger in the placebo group (fig 2, table 4). Patients in the placebo arm consumed a significantly higher mean number of loperamide tablets than patients in

Table 4 Effect of treatment on diarrhoea in the glutamine and placebo groups

\begin{tabular}{lccl}
\hline & Glutamine & Placebo & p Value $^{\star}$ \\
\hline All patients (n) & 29 & 33 & \\
Duration (mean (SD) days) & $1.5(2.4)$ & $2.8(3.0)$ & 0.07 \\
AUC (mean (SD)) & $1.9(3.1)$ & $4.5(5.9)$ & 0.09 \\
Loperamide (mean (SD) No of cps) & $0.4(1.1)$ & $2.6(3.5)$ & 0.002 \\
Only patients with diarrhoea (n) & 12 & 19 & \\
Duration (mean (SD) days) & $3.7(2.5)$ & $4.9(2.3)$ & 0.09 \\
AUC (mean (SD)) & $4.6(3.2)$ & $7.8(6.0)$ & 0.14 \\
Loperamide (mean (SD) No of cps) & $0.9(1.7)$ & $4.6(3.6)$ & 0.0006 \\
\hline
\end{tabular}

^Mann-Whitney test. the glutamine arm $(2.6 v 0.4 ; \mathrm{p}=0.002)$. Similar results were observed when the analysis was restricted to only those patients with diarrhoea.

Glutamine did not prevent severe stomatitis; in fact, grade $>2$ stomatitis was observed in five $(17 \%)$ patients who received glutamine and in seven $(21 \%)$ who received placebo. The mean duration of stomatitis was similar in the glutamine and placebo arms (4.2 and 3.4 days, respectively).

There was no difference in nausea, vomiting, or haematological toxicity between groups. Severe haematologic toxicity (grade 3-4 leucopenia) was observed in only two patients, one in each arm.

\section{Discussion}

The present study showed that an oral supplement of glutamine significantly reduced the degree of impairment of IA and IP in patients treated with FU/FA chemotherapy. This is the first randomised trial showing that oral glutamine may be effective in protecting the human intestinal mucosa from chemotherapy induced damage.

Our results are consistent with previous studies indicating that parenteral administration of glutamine has protective effects on the intestinal mucosa under different conditions. ${ }^{19}{ }^{20}$ Although a large and more recent study of glutamine enriched parenteral nutrition failed to confirm some of the benefits of the previous smaller trials, it showed a trend towards reduction in mortality in the glutamine group that would require an even larger randomised trial to be confirmed. ${ }^{30}$

To evaluate IA, we performed the urinary D-xylose test which reflects the absorptive surface of the small intestinal mucosa, ${ }^{31}$ because in a previous study it was positively affected by parenteral administration of glutamine dipeptides. $^{20}$

IP, a sensitive index of the morphological integrity of the small intestinal mucosa in a number of diseases, ${ }^{32}$ was evaluated using the cellobiose/mannitol test. This test had previously been proved to be useful in demonstrating an increase in IP in patients treated with FU/FA chemotherapy that was maximal 8-10 days after the start of therapy. ${ }^{33}$ A similar time scale was reported for increased gastrointestinal permeability in patients with advanced colon cancer after FU therapy. ${ }^{34}$

In the present study, chemotherapy with FU/FA reduced IA and increased IP in both the glutamine and placebo groups, consistent with a damaging effect of chemotherapy on the gut mucosa. However, changes in both IA and IP were significantly more marked in the placebo group. The effect of oral glutamine on IA is consistent with the results of Tremel and colleagues ${ }^{20}$ who showed higher urinary excretion of $\mathrm{D}$-xylose in patients receiving glutamine in their parenteral nutrition, although the size of the effect was lower in our study. For IP, our data are consistent with those of Van Der Hulst and colleagues ${ }^{19}$ who observed unchanged values for IP in patients receiving glutamine enriched parenteral nutrition compared with 
patients treated with standard parenteral nutrition whose IP increased during treatment.

Oral administration of glutamine is an inexpensive and convenient way of providing nutrient to patients with preserved oral intake. In fact, because glutamine is unstable during heat sterilisation and storage, ${ }^{35}$ it is included in amino acid solutions for parenteral use only in the form of dipeptides (either L-alanine-Lglutamine or glycil-L-glutamine) which are expensive and not widely available.

The $18 \mathrm{~g}$ daily dose of glutamine largely exceeds the normal dietary intake of $1 \mathrm{~g}$. This dose is well tolerated and is at the lower limit of the active dose range. ${ }^{37}$ This choice was also based on the consideration that larger volumes of water would have been required to dissolve higher doses, and this could have resulted in greater discomfort to patients, possibly suffering from chemotherapy induced nausea. As patients with malignant tumours of the gastrointestinal tract frequently have reductions in plasma, and perhaps the body pool of glutamine, ${ }^{38}$ treatment was started five days before the start of chemotherapy.

We did not find any difference in the incidence, duration, or severity of stomatitis in our patients. Although our trial was under powered for analysis of this issue, our finding is consistent with the negative results of Jebb and colleagues $^{39}$ who used a similar dose (16 g/day) of glutamine to prevent 5-FU+FA induced mucositis. However, a lower dose was effective in a more recent study. ${ }^{40}$ The authors explain this discrepancy on the basis of a longer duration of treatment and duration of local contact with the mouth, but the effect of glutamine on chemotherapy induced stomatitis remains to be established.

From a clinical point of view, the next step is to verify if the effects of oral glutamine on the IA and IP tests used in this study as a surrogate end point translate into a clinically relevant effect on chemotherapy induced diarrhoea. Indeed, the effect of glutamine on diarrhoea was only a secondary end point in our study that was under powered to identify small clinical differences. However, our clinical data suggest that glutamine can significantly reduce the duration and severity of diarrhoea. In view of this, two further issues deserve some comments. Firstly, the significantly higher consumption of loperamide in the placebo group, acting as rescue medication, could have diluted the differences in severity and duration of diarrhoea. In addition, as our study was limited to one cycle of chemotherapy, it is possible that longer exposure of the intestine to glutamine could have greater clinical efficacy.

Finally, the evidence that oral glutamine enhances the barrier function of the intestine could have implications in the treatment of diarrhoea from other causes associated with alterations of the small intestinal mucosa.

This work was partially supported by Ministero della Sanità and Regione Campania. The authors are grateful to Ms Silvana Corazza (Sister Olivia) for help in the care of patients.

Porazza (Sister Olivia) for help in the care of patients.
Presented in part at the Digestive Disease Week, Orlando,

Presented in part at the Digestive Disease Week, Orlando,
Florida, USA, May 16-19,1999 and published in abstract form in Gastroenterology 1999;116:A873.
1 Baskerville A, Batter-Hatton D. Intestinal lesions induced experimentally by methotrexate. $B r \quad \mathcal{f}$ Exp Pathol experimentally

2 Slavin RE, Dias MA, Saral R. Cytosine arabinoside-induced gastrointestinal toxic alterations in sequential chemotherapeutic protocols: a clinical-pathological study of 33 patients. Cancer 1978;42:1747-59.

3 Levin RJ. Anatomical and functional changes of the small intestine induced by 5-fluorouracil. F Physiol (Lond) 1968; 197:73-4P.

4 Siber GR, Mayer RJ, Levin MJ. Increased gastrointestinal absorption of large molecules in patients after 5 -fluorouracil therapy for metastatic colon carcinoma. Cancer Res 1980;40:3430-6.

5 de Roy van Zuidewijn DBW, Schillings PHM, Wobbes TH, et al. Morphometric analysis of the effects of antineoplastic drugs on mucosa of normal ileum and ileal anastomoses in drugs on mucosa of normal ileum and

6 Leichman CG, Fleming TR, Muggia FM, et al. Phase II study of fluorouracil and its modulation in advanced colo-
sel at Phase II rectal cancer: A Southwest Oncology Group study. F Clin rectal cancer: A Southwest

7 Petrelli N, Herrera L, Rustum Y, et al. A prospective randomized trial of 5-fluorouracil versus 5-fluorouracil and high-dose leucovorin versus 5-fluorouracil and methotrexate in previously untreated patients with advanced colorectal carcinoma. $\mathcal{F}$ Clin Oncol 1987;5:1559-65.

8 Labianca R, Pancera G, Luporini G. Factors influencing response rates for advanced colorectal cancer. Ann Oncol 1996;7:901-6.

9 Francini GP, Petriolo R, Lorenzini L, et al. Folinic acid and 5 -fluorouracil as adjuvant chemotherapy in colon cancer. Gastroenterology 1994;106:899-906.

10 Poon MA, O'Connell MJ, Moertel CG, et al. Biochemical modulation of fluorouracil: evidence of significant improvement of survival and quality of life in patients with ment of survival and quality of life in patients with 18 .

$11 \mathrm{Krebs} \mathrm{H}$. Glutamine metabolism in the animal body. In: Mora J, Palacios R, eds. Glutamine: metabolism, enzymology and regulation. New York: Academic Press, 1980:319-25.

12 Windmueller HG, Spaeth AE. Uptake and metabolism of plasma glutamine by the small intestine. F Biol Chem 1974; 249:5070-9.

13 Windmueller HG, Spaeth AE. Identification of ketone bodies and glutamine as the major respiratory fuels in vivo for postabsorptive rat small intestine. F Biol Chem 1978;253: 69-76.

14 Windmueller HG, Spaeth AE. Respiratory fuels and nitrogen metabolism in vivo in small intestine of fed rats: quantitative importance of glutamine, glutamate and quantitative importance of glutamine,

15 Baskerville A, Hambleton P, Benbough JE. Pathological features of glutaminase toxicities. Br f Exp Pathol 1980;61: 132-8.

16 Fox AD, Kripke SA, DePaula J, et al. Effect of a glutaminesupplemented enteral diet on methotrexate-induced enterocolitis. FPEN $\mathcal{F}$ Parenter Enteral Nutr 1988;12:325-31.

17 Klimberg VS, Souba WW, Dolson DJ, et al. Prophylactic glutamine protects the intestinal mucosa from radiation injury. Cancer 1990;66:62-8.

18 Souba WW. Glutamine: a key substrate for the splancnic bed. Anпи Rev Nutr 1991;11:283-308.

19 Van Der Hulst RRWJ, Van Kreel BK, Von Meyenfledt MF, et al. Glutamine and the preservation of gut integrity. Lancet 1993;334:1363-5.

20 Tremel H, Kienle B, Weilemann LS, et al. Glutamine dipeptide supplemented parenteral nutrition maintains intestinal function in the critically ill. Gastroenterology 1994;107: 1595-601.

21 Garner MLG, Samson RR, Heading RC. Changes in absorptive and peptide hydrolase activities in rat small intestine after administration of 5-fluorouracil. Clin Sci Mol Med 1978;54:411-18.

22 Juby LD, Rothwell J, Axon ATR. Cellobiose/mannitol sugar test. A sensitive tubeless test for coeliac disease: results on 1010 unselected patients. Gut 1989;30:476-80.

23 Olaison G. Abnormal intestinal permeability in Crohn's disease. A possible pathogenic factor. Scand $\mathcal{F}$ Gastroenterol 1990;25:321-8

24 Craig RM. D-xylose testing: a review. Gastroenterology 1988; 95:223-31.

25 US Department of Health and Human Services, National Institutes of Health, National Cancer Institute. Investigator's handbook: A manual for participants in clinical trials of tor's handbook: A manual for participants in clinical trials of
investigational agents sponsored by DCTD, NCI. Bethesda, investigational agents sponsored by DCTD, NCI

26 Peled Y, Doron O, Laufer H, et al. D-xylose absorption test: urine or blood? Dig Dis Sci 1991;36:188-92.

27 Corcoran A, Page IH. A method for the determination of mannitol in plasma and urine. Biol Chem 1947;170:16571

28 Strobel S, Brydon WG, Ferguson A. Cellobiose/mannitol sugar permeability test complements biopsy histopathology in clinical investigation of the jejunum. Gut 1984;25: $1241-6$

29 Matthews JNS, Altman DG, Campbell MJ, et al. Analysis of serial measurements in medical research. BMF 1990;300: 230-5.

30 Powell-Tuck J, Jamieson CP, Bettany GEA, et al. A doubleblind, randomised, controlled trial of glutamine supplementation in parenteral nutrition. Gut 1999;45:82-8.

31 Rolston DD, Matan VI. Xylose transport in human jejunum. Dig Dis Sci 1989;34:553-8. 
32 Bjarnason I, Macpherson AJ, Hollander D. Intestinal permeability: an overview. Gastroenterology 1995;108:156681 .

33 Daniele B, Secondulfo M, De Vivo R, et al. Effect of chemotherapy with 5 -fluorouracil on intestinal permeability and absorption of patients with advanced colorectal cancer. $\mathcal{F}$ Clin Gastroenterol (in press).

34 Siber GR, Mayer RJ, Levin MJ. Increased gastrointestinal absorption of large molecules in patients after 5-fluorouracil therapy for metastatic colon carcinoma Cancer Res 1980;40:3430-6.

35 Khan K, Hardy G, McElroy B, et al. The stability of L-glutamine in total parenteral nutrition solutions. Clin Nutr 1991;10:193-8.
36 Khan K, Elia M. Factors affecting the stability of L-glutamine in solution. Clin Nutr 1991;10:186-92.

37 Wilmore DW. Glutamine and the gut. Gastroenterology 1994;107:1885-6.

38 Kubota A, Meguid MM, Hitch DC. Amino acid profiles correlate diagnostically with organ site in three kinds of malignant tumors. Cancer 1992;69:2343-8.

39 Jebb SA, Osborne RJ, Maughan TS, et al. 5-fluorouracil and folinic acid-inducd mucositis: no effect of oral glutamine supplementation. Br f Cancer 1994;70:732-5.

40 Anderson PM, Schroeder MS, Skubitz KM. Oral glutamine reduces the duration and severity of stomatitis after cytotoxic cancer chemotherapy. Cancer 1998;83:1433-9. 